\title{
On the Complexity of Nucleolus Computation for Bipartite b-Matching Games *
}

\author{
Jochen Könemann \\ University of Waterloo \\ Waterloo, Ontario N2L 1A2 \\ jochen@uwaterloo.ca
}

\author{
Justin Toth \\ University of Waterloo \\ Waterloo, Ontario N2L 1A2 \\ wjtoth@uwaterloo.ca
}

\author{
Felix Zhou ${ }^{\dagger}$ \\ University of Waterloo \\ Waterloo, Ontario N2L 1A2 \\ cfzhou@uwaterloo.ca
}

December 27, 2022

\begin{abstract}
We explore the complexity of nucleolus computation in $b$-matching games on bipartite graphs. We show that computing the nucleolus of a simple $b$-matching game is $\mathcal{N} \mathcal{P}$-hard when $b \equiv 3$ even on bipartite graphs of maximum degree 7 . We complement this with partial positive results in the special case where $b$ values are bounded by 2 . In particular, we describe an efficient algorithm when a constant number of vertices satisfy $b_{v}=2$ as well as an efficient algorithm for computing the non-simple $b$-matching nucleolus when $b \equiv 2$.
\end{abstract}

\section{Introduction}

Consider a network of companies such that any pair with a pre-existing business relationship can enter into a deal that generates revenue, and at any given time every company has the capacity to fulfill a limited number of deals. This is an example of a scenario that can be modeled as a cooperative $b$-matching game.

A cooperative game is a pair $(N, \nu)$ where $N$ is a finite set of players and $\nu: 2^{N} \rightarrow \mathbb{R}$ is a value function which maps subsets of players, known as coalitions to a total value that their cooperation would generate. In the special case of simple cooperative b-matching games, we are given an underlying graph $G=(N, E)$, vertex values $b: N \rightarrow \mathbb{Z}_{+}$, and edge weights $w: E \rightarrow \mathbb{R}$. The set of players in the game corresponds to the vertices $N$, and $w(u v)$ denotes the value earned when $u, v \in N$ collaborate. For a coalition $S \subseteq N, \nu(S)$ corresponds to the maximum weight of a $b$-matching in $G[S]$ using each edge at most once. More formally, $\nu(S)$ is the optimal value of

$$
\begin{aligned}
& \max w(M) \\
|M \cap \delta(v)| & \leq b_{v} \\
M & \subseteq E[S] .
\end{aligned} \quad \forall v \in S
$$

\footnotetext{
${ }^{*}$ We acknowledge the support of the Natural Sciences and Engineering Research Council of Canada (NSERC). Cette recherche a été financée par le Conseil de recherches en sciences naturelles et en génie du Canada (CRSNG).

${ }^{\dagger}$ Present Address: Yale University, New Haven, CT 06511, felix.zhou@yale.edu
} 
On the other hand, in a non-simple cooperative b-matching game, $\nu(S)$ is modified to allow $M$ to be a multiset but we still require the the underlying set to be a subset of $E[S]$.

A central question in cooperative game theory is to study how the total revenue generated through the cooperation of the players is shared amongst the players themselves. An allocation $x \in \mathbb{R}^{N}$ is a vector whose entries indicate the value each player should receive. Not all allocations are equally desirable. Cooperative game theory gives us the language to model desirable allocations which capture notions such as fairness and stability.

An allocation $x \in \mathbb{R}^{N}$ is called efficient if its entries sum to $\nu(N)$; i.e., if $\sum_{i \in N} x_{i}:=x(N)=\nu(N)$. Efficiency stipulates that an allocation should distribute the total value generated by the grand coalition $N$. We say $x$ is an imputation if it is efficient and satisfies individual rationality: $x(i) \geq \nu(\{i\})$ for all $i$ in $N$. Individual rationality captures the notion that each player should be assigned at least the value they can earn on their own. In a $b$-matching game, $\nu(\{i\})=0$ and individual rationality simplifies to non-negativity.

The natural extension of individual rationality would be coalitional rationality, i.e. stipulating that for any coalition $S, x(S) \geq \nu(S)$. Allocations which satisfy such a property are said to lie in the core of the game. Core allocations can be considered highly stable in the sense that no subset of players can earn more value by deviating from the grand coalition.

The core is well-known to be non-empty in bipartite b-matching games [DIN99], but may be empty in general matching games. It is in fact known that the core of a matching game instance is non-empty if and only if the fractional matching linear program is integral [DIN99]. For example, the core of the matching instance given by an odd-cycle with unit weights is empty.

Since the core may be empty, we need a more robust solution concept. Given an allocation, we let $e(S, x):=$ $x(S)-\nu(S)$ be the excess of coalition $S \subseteq N$ with respect to $x$. Informally, the excess measures the satisfaction of coalition $S$ : the higher the excess of $S$, the more satisfied its players will be. We can rephrase the core as the set of all imputations where all coalitions have non-negative excess.

Instead of requiring all excesses to be non-negative, we can maximize the excess of the worst off coalitions. Consider the following linear program

$$
\begin{array}{rlrl}
\max \epsilon & & \left(P_{1}\right) \\
\text { s.t. } x(N) & =N & & \\
x(S) & \geq \nu(S)+\epsilon & & \forall S \subset N \\
x(i) & \geq \nu(\{i\}) & & \forall i \in N
\end{array}
$$

and let $\epsilon_{1}$ be its optimal solution. The least core is the set of allocations $x$ such that $\left(x, \epsilon_{1}\right)$ is optimal for $\left(P_{1}\right)$. The least core is always non-empty.

For $b$-matching games when the core is non-empty, the least core coincides with the core. When the core is empty, the least core tries to maximize the satisfaction of the coalitions who are worst off in the game. The least core, and by extension the core, both suffer from the fact that they are not in general unique. Furthermore, the least core does nothing to improve the satisfaction of coalitions which are not the worst off. This motivates the definition of the nucleolus, first introduced by Schmeidler [Sch69].

For an allocation $x$, we write $\theta(x) \in \mathbb{R}^{2^{N}-2}$ as the vector whose entries are $e(S, x)$ for all $\varnothing \neq S \subsetneq N$ sorted in non-decreasing order. This is a listing of the satisfactions of coalitions from worst off to best off. The nucleolus is defined as the allocation which lexicographically maximizes $\theta(x)$ over the imputation set. In a sense, the nucleolus is the most stable allocation. In Schmeidler's paper introducing the nucleolus, the author proved, among other things, that it is unique.

We now have sufficient terminology to state our main result, proven in Section 2.

Theorem 1.1 The problem of deciding whether an allocation is equal to the nucleolus of an unweighted bipartite 3-matching game is $\mathcal{N} \mathcal{P}$-hard, even in graphs of maximum degree $\%$ 
Kern and Paulusma posed the question of computing the nucleolus for general matching games as an open problem in 2003 [KP03]. In 2008, Deng and Fang conjectured this problem to be $\mathcal{N} \mathcal{P}$-hard [DF08]. This problem has been reaffirmed to be of interest in 2018 [Bir+18]. In 2020, Könemann, Pashkovich, and Toth proved the nucleolus of weighted matching games to be polynomial time computable [KPT20].

On one hand, computing the nucleolus of unweighted $b$-matching games when $b \geq 3$ is known to be $\mathcal{N} \mathcal{P}$-hard for general graphs [Bir+19]. However, the gadget graph in their hardness proof has many odd cycles. On the other hand, Bateni et al. provided an efficient algorithm to compute the nucleolus in bipartite $b$-matching games when one side of the bipartition is restricted to $b_{v}=1$ and the other side is unrestricted [Bat +10 ]. Thus it is a natural question whether the nucleolus of bipartite $b$-matching games is polynomial-time computable. Theorem 1.1 answers this question in the negative.

The basis of this result is a hardness proof for core separation in unweighted bipartite 3-matching games $[$ Bir +18$]$. However, extending this to a hardness proof of nucleolus computation requires significant technical innovation. Towards this end, we introduce a new problem in Section 2.2, a variant of the cubic subgraph problem, and prove that it is $\mathcal{N} \mathcal{P}$-hard. Then, in Section 2.1, we reduce the decision variant of nucleolus computation to our new problem, which yields the result.

In Section 3, we complement Theorem 1.1 with efficient algorithms to compute the nucleolus in two relevant cases when $b \leq 2$. Section 3.1 explores the scenario when only a constant number of vertices satisfy $b_{v}=2$ and Section 3.2 delves into the case when we relax the constraints to allow for non-simple $b$-matchings.

Theorem 1.2 Let $G$ be a simple bipartite graph with bipartition $N=A \cup \dot{B}$ and $k \geq 0$ a universal constant independent of $|N|$. Let $b \leq 2$ be some node-incidence capacity.

(i) Suppose $b_{v}=2$ for all $v \in A$ but $b_{v}=2$ for at most $k$ vertices of $B$, then the nucleolus of the simple weighted b-matching game on $G$ can be computed in polynomial time.

(ii) If $b \equiv 2$, then the nucleolus of the non-simple weighted b-matching game on $G$ can be computed in polynomial time.

We emphasize that for simple $b$-matching games, $\nu(\cdot)$ is defined by $b$-matchings where each edge is picked at most once. For non-simple $b$-matching games, we allow each edge to be picked multiple times.

\subsection{Related Work}

The assignment game, introduced by Shapley and Shubik [SS71], is the special case of simple $b$-matching games where $b$ is the all ones vector and the underlying graph is bipartite. This was generalized to matching games for general graphs by Deng, Ibaraki, and Nagamochi [DIN99]. Solymosi and Raghavan [SR94] showed how to compute the nucleolus in an unweighted assignment game. Kern and Paulusma [KP03] later gave a nucleolus computation algorithm for all unweighted matching games. Paulusma [Pau01] extended this result to all node-weighted matching games. An application of assignment games is towards cooperative procurement from the field of supply chain management [DK10].

The nucleolus is surprisingly ancient, appearing as far back in history as a scheme for bankruptcy division in the Babylonian Talmud [AM85]. Modern research interest in the nucleolus is not only based on its widespread application [BST05; Lem84], but also the complexity of computing the nucleolus, which seems to straddle the boundary between $\mathcal{P}$ and $\mathcal{N} \mathcal{P}$.

In a similar fashion to how we will define $b$-matching games, a wide variety of combinatorial optimization games can be defined [DIN99]. Here the underlying structure of the game is based on the optimal solution to some underlying combinatorial optimization problem. One might conjecture that the complexity of nucleolus computation for a combinatorial optimization game lies in the same class as its underlying combinatorial optimization problem. However, this is not in general true. For instance, nucleolus computation is known to be $\mathcal{N P}$-hard for network flow games [DFS09], weighted threshold games [Elk+07], and spanning tree games 
[FKK98; FKP00]. On the other hand, polynomial time algorithms are known for computing the nucleolus in special cases of network flow games [DFS09; PRB06], directed acyclic graph games [SS16; SFS17], spanning tree games [Gra+96; KSA00], b-matching games [Bat+10], fractional matching games [FKK01], weighted voting games [Elk+09], convex games [FKK01], and dynamic programming games [KT20].

One possible application of cooperative matching games is to network bargaining [Wil99; EK +12 ]. In this setting, a population of players are connected through an underlying social network. Each player engages in a profitable partnership with at most one of its neighbours and the profit must be shared between the participating players in some equitable fashion. Cook and Yamagishi [CY92] proposed a profit-sharing model that generalizes Nash's famous 2-player bargaining solution [NJ50] as well as validates empirical findings from the lab setting.

Both the pre-kernel and least-core are solution concepts which contain the nucleolus. It is well-known that the pre-kernel of a cooperative game may be non-convex and even disconnected [Kop67; Ste68]. Nonetheless, Faigle, Kern, and Kuipers showed how to compute a point in the intersection of the pre-kernel and least-core in polynomial time given a polynomial time oracle to compute the minimum excess coalition for a given allocation [FKK01]. The authors later refined their result to compute a point within the intersection of the core and lexicographic kernel [FKK06], a set which also contains the nucleolus.

The complexity of computing the nucleolus of $b$-matching games remained open for bipartite graphs, and for b-matching games where $b \leq 2$. In Theorem 1.1, we show that the former is indeed $\mathcal{N} \mathcal{P}$-hard to compute and give an efficient algorithm for a special case of the latter in Section 3. Some positive results about the core were proved for non-bipartite graphs in [Bir+18].

\subsection{The Kopelowitz Scheme}

A more computational definition of the nucleolus is provided by the Kopelowitz Scheme [Kop67]. Recall the linear program $\left(P_{1}\right)$ and let $\epsilon_{1}$ be its optimal value. Write $\mathcal{S}:=2^{N} \backslash\{\varnothing, N\}$ to denote the set of all non-trivial coalitions. Finally, put

$$
\mathcal{S}_{1}:=\left\{S \in \mathcal{S}: x(S)=\nu(S)+\epsilon_{1} \text { for all optimal solutions }\left(x, \epsilon_{1}\right)\right\} .
$$

We say $\mathcal{S}_{1}$ are the coalitions which are fixed in $\left(P_{1}\right)$.

For $\ell \geq 2$, let $\left(P_{\ell}\right)$ be the linear program

$$
\begin{gathered}
\max \epsilon \\
x(N)=\nu(N) \\
x(S)=\nu(S)+\epsilon_{i} \\
x(S) \geq \nu(N)+\epsilon
\end{gathered}
$$

Recursively, we set

$$
\mathcal{S}_{\ell}:=\left\{S \in \mathcal{S}: \forall i \leq \ell-1, S \notin \mathcal{S}_{i}, x(S)=\nu(S)+\epsilon_{\ell} \text { for all optimal solutions }\left(x, \epsilon_{\ell}\right) \text { to }\left(P_{\ell}\right)\right\} .
$$

These are the coalitions which are fixed in $\left(P_{\ell}\right)$ but not in any $\left(P_{i}\right)$ for $i \leq \ell-1$. This hierarchy of linear programs terminates when the dimension of the feasible region becomes 0 , at which point the unique feasible solution is the nucleolus [DM65].

Directly solving each $\left(P_{\ell}\right)$ requires solving a linear program with an exponential number of constraints in terms of the number of players and hence takes exponential time with respect to the input ${ }^{1}$. Moreover, the

\footnotetext{
${ }^{1}$ Cooperative games we are interested in have a compact representation roughly on the order of the number of players. For example $b$-matching games can be specified by a graph, $b$-values and edge weights rather than explicitly writing out $\nu$.
} 
best general bound on the number of linear programs we must solve until we obtain a unique solution is the naive exponential bound $O\left(2^{|N|}\right)$. However, we are still able to use the Kopelowitz Scheme as a way to characterize the nucleolus in the proof of Theorem 1.1.

One way of solving exponentially sized linear programs is to utilize the polynomial time equivalence of optimization and separation [Kha79]. That is, to develop a separation oracle and employ the ellipsoid method. For our positive results, we will take this route.

Indeed, we will develop a polynomial-size formulation of each $\left(P_{\ell}\right)$ by pruning unnecessary constraints. Not only does this enable us to solve each $\left(P_{\ell}\right)$ in polynomial time, but we also reduce the number of iterations to a polynomial of the input size since at least one inequality constraint is changed to an equality constraint per iteration.

It is of interest to consider a variation of the Kopelowitz Scheme by Maschler [MPS79]. In this variation, the author defines $\mathcal{S}_{\ell}$ as

$$
\mathcal{S}_{\ell}:=\left\{S \in \mathcal{S}: \forall i \leq \ell-1, S \notin \mathcal{S}_{i}, \exists c_{S} \in \mathbb{R} \text { such that } x(S)=c_{S} \text { for all optimal solutions } x \text { of }\left(P_{\ell}\right)\right\} .
$$

This way, at least 1 equality constraint is added to $\left(P_{\ell+1}\right)$ which is linearly independent of all equality constraints in $\left(P_{\ell}\right)$. Hence the feasible region decreases by at least 1 dimension per iteration and there are at most $|N|$ iterations before termination.

\section{Hardness}

We consider $b$-matching games for $b \equiv 3$ and uniform weights. The goal of the this section is to prove Theorem 1.1.

The idea of the proof is inspired the hardness proof of core separation employed in [Bir +18$]$ and the hardness proof in [DFS09]. We reduce the problem into a variation of Cubic SubGraph which is $\mathcal{N} \mathcal{P}$-hard [Ple84] through a careful analysis of several iterations of the Kopelowitz Scheme. However, it is not clear that our variation of CUBIC SUBgRAPH is $\mathcal{N} \mathcal{P}$-hard and we significantly extend the techniques from [Ple84] to prove its hardness.

Problem 1 (Two from Cubic Subgraph) Let $G$ be an arbitrary graph. Decide if $G$ contains a subgraph $H$ (not necessarily induced) such that there are $u \neq v \in V(H)$ satisfying

$$
\operatorname{deg}_{H}(w)= \begin{cases}2, & w \in\{u, v\} \\ 3, & \text { else }\end{cases}
$$

for all $w \in V(H)$. We say that $H$ is a Two from Cubic Subgraph.

Theorem 2.1 Two from Cubic Subgraph is $\mathcal{N} \mathcal{P}$-hard, even in bipartite graphs of maximum degree 4.

This theorem is proven in Section 2.2 and is crucial to our proof of Theorem 1.1 in Section 2.1.

\subsection{The Reduction}

Hereinafter, $G=(N, E)$ is a bipartite instance of Two From Cubic Subgraph. We assume that $E \neq \varnothing$ so that $|N| \geq 2$. Take $G^{*}:=\left(N^{*}, E^{*}\right)$ to be the following bipartite gadget graph depicted in Figure 1, initially proposed in [Bir+18]: For each original vertex $u \in N$, create 5 new vertices $v_{u}, w_{u}, x_{u}, y_{u}, z_{u}$. Then, define $N^{*}:=N \cup\left\{v_{u}, w_{u}, x_{u}, y_{u}, z_{u}: u \in N\right\}$. To obtain $E^{*}$ from $E$, we add edges until $\left(\left\{u, v_{u}, w_{u}\right\},\left\{x_{u}, y_{u}, z_{u}\right\}\right)$ is a $K_{3,3}$ subgraph for every $u \in N$. Notice that $E^{*} \backslash E$ forms a maximum 3-matching in $G^{*}$ with cardinality $\frac{3}{2}\left|N^{*}\right|$. 


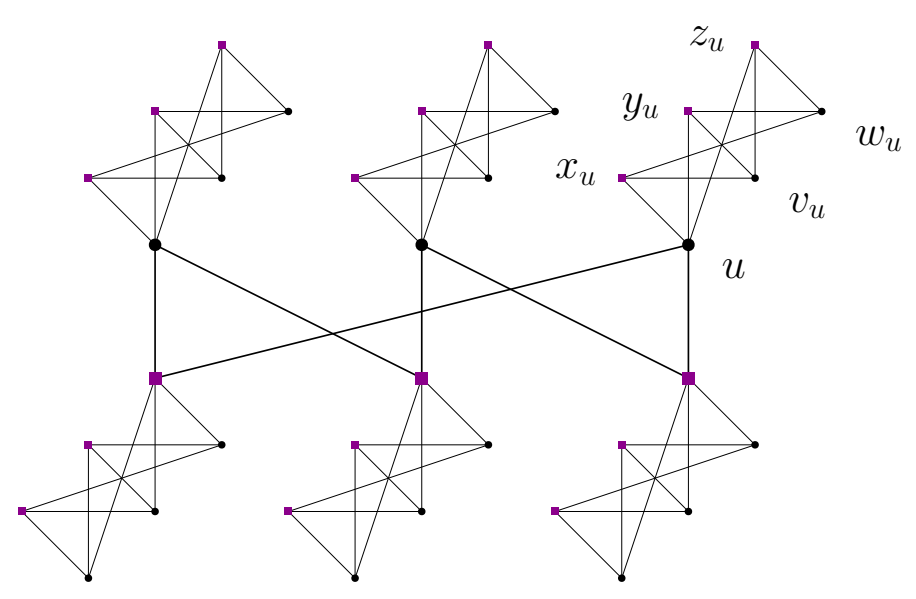

Figure 1: The gadget graph from $[\mathrm{Bir}+18]$.

In Figure 1, the bigger vertices with bolded edges indicate the original graph and the smaller vertices with thinner edges were added to obtain the gadget graph. The square and circular vertices depict a bipartition of $G^{*}$. Observe that the maximum degree of $G^{*}$ is the maximum degree of $G$ plus 3 .

For each $u \in N$, define $T_{u}:=\left\{v_{u}, w_{u}, x_{u}, y_{u}, z_{u}\right\}$ as well as $V_{u}:=T_{u} \cup\{u\}$. We say that $T_{u}$ are the gadget vertices of $u$ and $V_{u}$ is the complete gadget of $u$. Remark that each $V_{u}$ induces a connected component within the maximum 3-matching $E^{*} \backslash E$ on $G^{*}$.

Let $\Gamma=\left(N^{*}, \nu\right)$ be the unweighted 3-matching game on $G^{*}$.

In Lemma 2.3 , we show that if no two from cubic subgraph of $G$ exists, then the nucleolus is precisely $x^{*} \equiv \frac{3}{2}$. Conversely, we prove in Lemma 2.4 that the existence of a two from cubic subgraph implies that $x^{*}$ cannot be the nucleolus. The proof of Theorem 1.1 follows from the above lemmas and the hardness of TwO FROM Cubic Subgraph proven in Section 2.2. Remark that the degree bound follows from the degree bound in Theorem 2.1 and the fact that our gadget graph increases the maximum degree of the original graph by 3 .

Lemma 2.2 Let $M$ be a maximum 3-matching in $G^{*}$. Let $\mathcal{C}$ be the set of connected components of $G^{*}[M]$. Then for all core allocations $x$ and every component $C \in \mathcal{C}, x(C)=\nu(C)$.

Proof: Observe that

$$
x\left(N^{*}\right) \geq x(M)=\sum_{C \in \mathcal{C}} x(C) \geq \sum_{C \in \mathcal{C}} \nu(C)=\sum_{C \in \mathcal{C}}|M \cap E(C)|=|M|=\nu\left(N^{*}\right)
$$

with the first inequality following from the fact that $x \geq 0$ and the second inequality following from the assumption that $x$ is in the core.

Lemma 2.3 If $G$ does not contain a two from cubic subgraph, the uniform allocation $x^{*} \equiv \frac{3}{2}$ is the nucleolus of $\Gamma$.

Proof: We argue using the Kopelowitz Scheme. Put $\left(P_{k}\right)$ as the $k$-th LP in the Kopelowitz Scheme.

We check through computation that for all $u \in N$ and $S \subsetneq V_{u}, e\left(S, x^{*}\right) \geq \frac{3}{2}$. See Table 1 .

Let $\epsilon_{1}$ be the optimal objective value to $\left(P_{1}\right)$. We claim that $\epsilon_{1}=0$. By core non-emptiness, we have $\epsilon_{1} \geq 0$. Since $E \neq \varnothing$, we can choose $u \in N$ so that $V_{u} \subsetneq N$. But $V_{u}$ is a connected component of the maximum 3 -matching $E^{*} \backslash E$. Thus by Lemma $2.2, V_{u}$ is a coalition for which $e\left(V_{u}, x\right)=0$ for all core allocations $x$. It follows that $\epsilon_{1}=0$ and the set of optimal solutions to $\left(P_{1}\right)$ is precisely the core. 


\begin{tabular}{|c|c|c|r|}
\hline$\left|S \cap\left\{u, v_{u}, w_{u}\right\}\right|$ & $\left|S \cap\left\{x_{u}, y_{u}, z_{u}\right\}\right|$ & $\nu(S)$ & $e\left(S, x^{*}\right)$ \\
\hline \hline 0 & 1 & 0 & $\frac{3}{2}$ \\
0 & 2 & 0 & 3 \\
0 & 3 & 0 & $\frac{9}{2}$ \\
1 & 0 & 0 & $\frac{3}{2}$ \\
1 & 1 & 1 & 2 \\
1 & 2 & 2 & $\frac{5}{2}$ \\
1 & 3 & 3 & 3 \\
2 & 0 & 0 & 3 \\
2 & 1 & 2 & $\frac{5}{2}$ \\
2 & 2 & 4 & 2 \\
2 & 3 & 6 & $\frac{3}{2}$ \\
3 & 0 & 0 & $\frac{9}{2}$ \\
3 & 1 & 3 & 3 \\
3 & 2 & 6 & $\frac{3}{2}$ \\
\hline
\end{tabular}

Table 1: The excess computation for Lemma 2.3 when $x^{*} \equiv \frac{3}{2}, S \subsetneq V_{u}$.

We now claim that

$$
\mathcal{S}_{1}=\left\{S \subseteq N^{*}: S=\bigcup_{u \in S \cap N} V_{u}\right\} .
$$

These are the unions of complete gadgets.

Let $x$ be an optimal solution to $\left(P_{1}\right)$ (core allocation). Clearly, if $S$ is a union of complete gadgets, then $e\left(S, x^{*}\right)=0$ due to Lemma 2.2. This shows the reverse inclusion in Equation (5). Notice that $\nu(S) \leq \frac{3}{2}|S|=$ $x^{*}(S)$ by the definition of a 3-matching, so $x^{*}$ is an optimal solution to $\left(P_{1}\right)$ and we may assume that $x=x^{*}$.

We claim that

$$
\forall S \notin \mathcal{S}_{1}, e\left(S, x^{*}\right) \geq \frac{3}{2} .
$$

This shows that if $S$ is not a union of complete gadgets, then there is some optimal solution of $\left(P_{1}\right)$ for which $S$ is not fixed in $\left(P_{1}\right)$ and hence $S \notin \mathcal{S}_{1}$. Thus the inclusion in Equation (5) would hold.

Equation (6) is true if $S=\{u\}$ for some $u \in N^{*}$. If $S \subseteq N$ with $|S| \geq 2$, then $\nu(S) \leq \frac{3}{2}|S|-2$. Otherwise, the edges of a maximum 3-matching in $G[S]$ induce a two from cubic subgraph. Thus $e\left(S, x^{*}\right) \geq$ $\frac{3}{2}|S|-\left(\frac{3}{2}|S|-2\right) \geq 2$.

It remains to consider the case when there is some $u \in N$ such that $T_{u} \cap S \neq \varnothing$. The argument here is similar to the one employed in [Bir+18]: First suppose $S \cap V_{u}=V_{u}$ and remark that there is a maximum 3-matching in $G^{*}[S]$ where all edges adjacent to $u$ are contained in the edge set induced by $V_{u}$. Thus deleting such edges yields a 3 -matching in $G^{*}\left[S \backslash V_{u}\right]$.

$$
\begin{aligned}
& e\left(S \backslash V_{u}, x^{*}\right) \\
& \leq e\left(S, x^{*}\right)-x^{*}\left(V_{u}\right)+\left|E^{*}\left(V_{u}\right)\right| \quad \nu\left(S \backslash V_{u}\right) \geq \nu(S)-\left|E^{*}\left(V_{u}\right)\right| \\
& =e\left(S, x^{*}\right)-9+9 \\
& =e\left(S, x^{*}\right) .
\end{aligned}
$$


We can remove as many of these complete gadgets from $S$ as possible to obtain some coalition $S^{\prime}$ with $e\left(S^{\prime}, x^{*}\right) \leq e\left(S, x^{*}\right)$. It suffices then to lower bound $e\left(S^{\prime}, x^{*}\right)$.

If $S^{\prime}=\varnothing$, then $S \in \mathcal{S}_{1}$ by definition. In addition, if $S^{\prime} \subseteq N$, there is again nothing to prove. Thus there must be some $u^{\prime} \in S^{\prime}$ such that $\left|T_{u^{\prime}} \cap S^{\prime}\right| \geq 1$ and $S^{\prime} \cap V_{u^{\prime}} \neq V_{u^{\prime}}$.

Suppose now that $\left|S^{\prime} \cap T_{u^{\prime}}\right| \leq 4$. Remark that if $M$ is a 3-matching in $G^{*}\left[S^{\prime}\right]$, then $M \backslash E^{*}\left(S^{\prime} \cap T_{u^{\prime}} \cup\left\{u^{\prime}\right\}\right)$ is a 3-matching in $G^{*}\left[S^{\prime} \backslash T_{u^{\prime}}\right]$.

$$
\begin{aligned}
& e\left(S^{\prime} \backslash T_{u^{\prime}}, x^{*}\right) \\
& \leq e\left(S^{\prime}, x^{*}\right)-x^{*}\left(S^{\prime} \cap T_{u^{\prime}}\right)+\left|E^{*}\left(S^{\prime} \cap T_{u^{\prime}} \cup\left\{u^{\prime}\right\}\right)\right| \quad \nu\left(S^{\prime} \backslash T_{u^{\prime}}\right) \geq \nu\left(S^{\prime}\right)-\left|E^{*}\left(S^{\prime} \cap T_{u^{\prime}} \cup\left\{u^{\prime}\right\}\right)\right| \\
& =e\left(S^{\prime}, x^{*}\right)-\frac{3}{2}\left|S^{\prime} \cap T_{u^{\prime}}\right|+\left|E^{*}\left(S^{\prime} \cap T_{u^{\prime}} \cup\left\{u^{\prime}\right\}\right)\right| \\
& \leq e\left(S^{\prime}, x^{*}\right) .
\end{aligned}
$$

Finally, if $\left|S^{\prime} \cap T_{u^{\prime}}\right|=5$, we are required to have $u^{\prime} \notin S^{\prime}$. If $M$ is a 3-matching in $G^{*}\left[S^{\prime}\right]$, then $M \backslash E^{*}\left(S^{\prime} \cap T_{u^{\prime}}\right)$ is a 3 -matching in $G^{*}\left[S^{\prime} \backslash T_{u^{\prime}}\right]$.

$$
\begin{aligned}
& e\left(S^{\prime} \backslash T_{u^{\prime}}, x^{*}\right) \\
& \leq e\left(S^{\prime}, x^{*}\right)-x^{*}\left(S^{\prime} \cap T_{u^{\prime}}\right)+\nu\left(T_{u^{\prime}}\right) \quad \nu\left(S^{\prime} \backslash T_{u^{\prime}}\right) \geq \nu\left(S^{\prime}\right)-\nu\left(T_{u^{\prime}}\right) \\
& =e\left(S^{\prime}, x^{*}\right)-5 \cdot \frac{3}{2}+6 \\
& \leq e\left(S^{\prime}, x^{*}\right) .
\end{aligned}
$$

We may thus again repeatedly remove vertices of $N^{*} \backslash N$ until we arrive back at the base case of $S^{\prime} \subseteq N$.

So Equation (6) holds as all other coalitions have strictly greater excess with respect to $x^{*}$.

We now argue that $\epsilon_{2}=\frac{3}{2}$. Observe $\nu\left(N^{*}\right)=\frac{3}{2}\left|N^{*}\right|$ implies that $\min _{a \in N^{*}} x(a) \leq \frac{3}{2}$ for any allocation $x$ in the core and thus also for feasible solutions to $\left(P_{2}\right)$ as well as the nucleolus. It follows that $\epsilon_{2} \leq \frac{3}{2}$. But Equation (6) shows that this upper bound is attained by $x^{*}$.

For all feasible solutions $x$ to $\left(P_{2}\right), x(a) \geq \frac{3}{2}$ for all $a \in N^{*}$. But we cannot have some $x(a)>\frac{3}{2}$, or else $x\left(N^{*}\right)>\frac{3}{2}\left|N^{*}\right|$ and $x$ would not be an allocation. Since the singleton coalitions are fixed in $\left(P_{2}\right)$, it must be that $x^{*} \equiv \frac{3}{2}$ is the nucleolus.

Lemma 2.4 If $G$ contains a two from cubic subgraph, then the nucleolus of the gadget graph is not $x^{*} \equiv \frac{3}{2}$.

Proof: Suppose that $G$ contains a two from cubic subgraph. We will show that $x^{*} \equiv \frac{3}{2}$ is not an optimal solution to $\left(P_{2}\right)$. Recall that the nucleolus is necessarily an optimal solution to each LP in the Kopelowitz Scheme. This would thus yield the desired result.

Let us introduce a parameter as follows:

$$
\Delta:= \begin{cases}0, & G \text { contains a cubic subgraph } \\ 1, & G \text { contains a two from cubic subgraph but no cubic subgraphs }\end{cases}
$$

Let $N^{\prime} \subseteq N$ be the vertices in the cubic subgraph or the vertices of the two from cubic subgraph if no cubic subgraph exists. Then

$$
e\left(N^{\prime}, x^{*}\right)=\frac{3}{2}\left|N^{\prime}\right|-\left(\frac{3}{2}\left|N^{\prime}\right|-\Delta\right)=\Delta .
$$

In particular, the minimum excess over all coalitions in $\mathcal{S}$ is at most $\Delta$.

For $0<\delta<\frac{1}{2}$, define

$$
x_{\delta}(a):= \begin{cases}\frac{3}{2}+\delta, & a \in N \\ \frac{3}{2}-\frac{\delta}{5}, & a \in N^{*} \backslash N\end{cases}
$$




\begin{tabular}{|c|c|c|c|c|}
\hline$|S \cap\{u\}|$ & $\left|S \cap\left\{v_{u}, w_{u}\right\}\right|$ & $\left|S \cap\left\{x_{u}, y_{u}, z_{u}\right\}\right|$ & $\nu(S)$ & $e\left(S, x^{*}\right)$ \\
\hline 0 & 0 & 1 & 0 & $\frac{3}{2}-\frac{\delta}{5}$ \\
\hline 0 & 0 & 2 & 0 & $3-\frac{2 \delta}{5}$ \\
\hline 0 & 0 & 3 & 0 & $\frac{9}{2}-\frac{3 \delta}{5}$ \\
\hline 0 & 1 & 0 & 0 & $\frac{3}{2}-\frac{\delta}{5}$ \\
\hline 0 & 1 & 1 & 1 & $2-\frac{2 \delta}{5}$ \\
\hline 0 & 1 & 2 & 2 & $\frac{5}{2}-\frac{3 \delta}{5}$ \\
\hline 0 & 1 & 3 & 3 & $3-\frac{4 \delta}{5}$ \\
\hline 0 & 2 & 0 & 0 & $3-\frac{2 \delta}{5}$ \\
\hline 0 & 2 & 1 & 2 & $\frac{5}{2}-\frac{2 \delta}{5}$ \\
\hline 0 & 2 & 2 & 4 & $2-\frac{4 \delta}{5}$ \\
\hline 0 & 2 & 3 & 6 & $\frac{3}{2}-\delta$ \\
\hline 1 & 0 & 0 & 0 & $\frac{3}{2}+\delta$ \\
\hline 1 & 0 & 1 & 1 & $2+\frac{4 \delta}{5}$ \\
\hline 1 & 0 & 2 & 2 & $\frac{5}{2}+\frac{3 \delta}{5}$ \\
\hline 1 & 0 & 3 & 3 & $3+\frac{2 \delta}{5}$ \\
\hline 1 & 1 & 0 & 0 & $3+\frac{4 \delta}{5}$ \\
\hline 1 & 1 & 1 & 2 & $\frac{5}{2}+\frac{3 \delta}{5}$ \\
\hline 1 & 1 & 2 & 4 & $2+\frac{2 \delta}{5}$ \\
\hline 1 & 1 & 3 & 6 & $\frac{3}{2}+\frac{\delta}{5}$ \\
\hline 1 & 2 & 0 & 0 & $\frac{9}{2}+\frac{3 \delta}{5}$ \\
\hline 1 & 2 & 1 & 3 & $3+\frac{2 \delta}{5}$ \\
\hline 1 & 2 & 2 & 6 & $\frac{3}{2}+\frac{\delta}{5}$ \\
\hline
\end{tabular}

Table 2: The excess computation for Lemma 2.4 when $x_{\delta}, S \subsetneq V_{u}$. 
We check by computation in Table 2 that

$$
\forall u \in N, \forall S \subsetneq V_{u}, e\left(S, x_{\delta}\right) \geq \frac{3}{2}-\delta
$$

The coalitions with minimum excess among such coalitions is $S=T_{u}$ for some $u \in N$.

We claim that $\epsilon_{1}=0$ and

$$
\mathcal{S}_{1}=\left\{S \subseteq N^{*}: S=\bigcup_{u \in S \cap N} V_{u}\right\} .
$$

is again the union of complete gadgets. The fact that $\epsilon_{1}=0$ is clear from our previous lemma. Moreover, it is clear from our prior work that the unions of complete gadgets must be fixed in $\left(P_{1}\right)$. We need only show that $e\left(S, x_{\delta}\right)>0$ if $S$ is not a union of complete gadgets. This would show that if $S$ is not a union of complete gadgets, then there is some allocation (in particular $x_{\delta}$ ) for which $S$ is not fixed in $\left(P_{1}\right)$.

If $S=\{a\}$ for some $a \in N^{*}$, then $e\left(S, x_{\delta}\right) \geq \frac{3}{2}-\frac{\delta}{5}>0$.

When $S \subseteq N$, we have

$$
\begin{aligned}
e\left(S, x_{\delta}\right) & =x_{\delta}(S)-\nu(S) \\
& \geq\left(\frac{3}{2}+\delta\right)|S|-\left(\frac{3}{2}|S|-\Delta\right) \\
& =\delta|S|+\Delta \\
& >0 .
\end{aligned}
$$

Suppose now that there is some $u \in N$ such that $S \cap T_{u} \neq \varnothing$. Once again, if $S \cap V_{u}=V_{u}, e\left(S \backslash V_{u}, x_{\delta}\right) \leq$ $e\left(S, x_{\delta}\right)-9+9=e\left(S, x_{\delta}\right)$. We can thus remove all complete gadgets from $S$ to obtain another coalition $S^{\prime}$. If $S^{\prime}=\varnothing$, then $S \in \mathcal{S}_{1}$. Similar to before, if $S^{\prime} \subseteq N$, we are back at the base case.

We proceed assuming there is some $u^{\prime} \in S^{\prime}$ such that $\left|S^{\prime} \cap T_{u^{\prime}}\right| \geq 1$. Observe that $\nu\left(S^{\prime}\right) \leq \nu\left(S^{\prime} \backslash T_{u^{\prime}}\right)+$ $\nu\left(S^{\prime} \cap V_{u^{\prime}}\right)$. This is because any maximum 3-matching on $S^{\prime}$ is a disjoint union of 3-matchings on $S^{\prime} \backslash T_{u^{\prime}}$ and $S^{\prime} \cap V_{u^{\prime}}$.

Suppose $u^{\prime} \in S^{\prime}$. We must have $\left|S^{\prime} \cap T_{u^{\prime}}\right| \leq 4$.

$$
\begin{aligned}
e\left(S^{\prime}, x_{\delta}\right) & =x_{\delta}\left(S^{\prime} \backslash T_{u^{\prime}}\right)+x_{\delta}\left(S^{\prime} \cap V_{u^{\prime}}\right)-x_{\delta}\left(u^{\prime}\right)-\nu\left(S^{\prime}\right) \\
& \geq x_{\delta}\left(S^{\prime} \backslash T_{u^{\prime}}\right)-\nu\left(S^{\prime} \backslash T_{u^{\prime}}\right)+\left[x_{\delta}\left(S^{\prime} \cap V_{u^{\prime}}\right)-x_{\delta}\left(u^{\prime}\right)\right]-\nu\left(S^{\prime} \cap V_{u^{\prime}}\right) \\
& \geq e\left(S^{\prime} \backslash T_{u^{\prime}}, x_{\delta}\right)+\left|S \cap T_{u^{\prime}}\right|\left(\frac{3}{2}-\frac{\delta}{5}\right)-\left|E^{*}\left(S^{\prime} \cap V_{u^{\prime}}\right)\right| \\
& \geq e\left(S^{\prime} \backslash T_{u^{\prime}}, x_{\delta}\right)-\frac{4}{5} \delta .
\end{aligned}
$$

Suppose now that $u^{\prime} \notin S^{\prime}$. In this case,

$$
\begin{aligned}
e\left(S^{\prime}, x_{\delta}\right) & =x_{\delta}\left(S^{\prime} \backslash T_{u^{\prime}}\right)+x_{\delta}\left(S^{\prime} \cap T_{u^{\prime}}\right)-\nu\left(S^{\prime}\right) \\
& \geq x_{\delta}\left(S^{\prime} \backslash T_{u^{\prime}}\right)-\nu\left(S^{\prime} \backslash T_{u^{\prime}}\right)+x_{\delta}\left(S^{\prime} \cap T_{u^{\prime}}\right)-\nu\left(S^{\prime} \cap T_{u^{\prime}}\right) \\
& =e\left(S^{\prime} \backslash T_{u^{\prime}}, x_{\delta}\right)+e\left(S^{\prime} \cap T_{u^{\prime}}, x_{\delta}\right) \\
& \geq e\left(S^{\prime} \backslash T_{u^{\prime}}, x_{\delta}\right)+\left(\frac{3}{2}-\delta\right)
\end{aligned}
$$

by Equation (8) 
By repeatedly removing vertices of $N^{*} \backslash N$, we see that

$$
\begin{aligned}
e\left(S^{\prime}, x_{\delta}\right) & \geq e\left(S^{\prime} \cap N, x_{\delta}\right)+\sum_{u \in S^{\prime} \cap N: S^{\prime} \cap T_{u} \neq \varnothing}\left[-\frac{4}{5} \delta\right]+\sum_{u \in N \backslash S^{\prime}: S^{\prime} \cap T_{u} \neq \varnothing}\left[\frac{3}{2}-\delta\right] \\
& \geq \delta\left|S^{\prime} \cap N\right|+\Delta-\left|S^{\prime} \cap N\right|\left(\frac{4}{5} \delta\right)+\left|\left\{u \in N \backslash S^{\prime}: S^{\prime} \cap T_{u} \neq \varnothing\right\}\right|\left(\frac{3}{2}-\delta\right) \\
& =\frac{\delta}{5}\left|S^{\prime} \cap N\right|+\Delta+\left|\left\{u \in N \backslash S^{\prime}: S^{\prime} \cap T_{u} \neq \varnothing\right\}\right|\left(\frac{3}{2}-\delta\right) \\
& \geq \frac{\delta}{5}+\Delta .
\end{aligned}
$$

The last inequality follows from the assumption that $S^{\prime} \neq \varnothing$. In particular, at least one of $S^{\prime} \cap N$ or $\left\{u \in N \backslash S^{\prime}: S^{\prime} \cap T_{u} \neq \varnothing\right\}$ is non-empty. This shows that $\epsilon_{1}=0$ is indeed the optimal solution to $\left(P_{1}\right)$. Moreover, $\mathcal{S}_{1}$ is again the union of complete gadgets.

As an immediate corollary to the proof, $\epsilon_{2} \geq \frac{\delta}{5}+\Delta>\Delta$. Recall there was a coalition $N^{\prime} \subseteq N$ satisfying Equation (7). It follows that $x^{*} \equiv \frac{3}{2}$ is not an optimal solution to $\left(P_{2}\right)$ and therefore cannot be the nucleolus. $\square$

\subsection{Two from Cubic Subgraph}

In this subsection, we prove that Two From CUBic SubgraPh, from which we reduce to nucleolus testing, is $\mathcal{N} \mathcal{P}$-hard.

Problem 2 (Exact Cover by 3-Sets) Let a ground set $X=\left\{a_{1}, a_{2}, \ldots, a_{3 k}\right\}$ and a collection of 3element subsets of $X S=\left\{S_{1}, S_{2}, \ldots, S_{t}\right\}$ be given.

Decide if there is a subcollection $Y \subseteq S$ where each element $a_{i} \in X$ is included in exactly one subset $S_{j} \in Y$.

It is well known that ExACT Cover BY 3-SETS is $\mathcal{N} \mathcal{P}$-hard, even if every element of the ground set belongs to exactly three subsets [Gon85]. We reduce Exact Cover by 3-Sets to Two from Cubic Subgraph.

The idea is to construct two parallel instances of the bipartite gadget graph from [Ple84]. We will then focus on any "non-trivial" two from cubic subgraphs.

\subsubsection{Step I}

Let $X, S$ be an instance of the EXACT COVER BY 3-SETS where every element of the ground set belongs to exactly three subsets. Create the bipartite graph $G_{0}$ with bipartition $X \dot{\cup} S$, where $a_{i} S_{j} \in E\left(G_{0}\right) \Longleftrightarrow a_{i} \in$ $S_{j}$. The problem is reformulated as follows: Does there exist a subgraph with vertex set $X \cup S^{\prime}$ such that every vertex of $X$ has degree 1 and each vertex of $S^{\prime}$ has degree 3 ? Notice we require the entire ground set to be included in the subgraph vertex set.

\subsubsection{Step II}

Add $7 k$ new vertices to $G_{0}, b_{1}, b_{2}, \ldots, b_{7 k}$, as follows. Each $b_{i}, 1 \leq i \leq 3 k$ is adjacent to $a_{i}$ and $b_{3 k+i}$. If $i>1$, then $b_{i}$ is also adjacent to $b_{3 k+i-1}$. Otherwise, $i=1$ and $b_{i}$ is also adjacent to $b_{6 k}$. Finally, each $b_{6 k+j}, 1 \leq j \leq k$, is adjacent to $b_{3 k+3 j-2}, b_{3 k+3 j-1}, b_{3 k+3 j}$.

See Figure 2. The square and circular vertices depict a bipartition of $G_{1}$.

Define the following tripartition of the newly added vertices $B_{1}:=\left\{b_{i}: 1 \leq i \leq 3 k\right\}, B_{2}:=\left\{b_{3 k+i}: 1 \leq i \leq\right.$ $3 k\}$, and $B_{3}:=\left\{b_{6 k+i}: 1 \leq i \leq k\right\}$.

Let us refer to this graph as $G_{1}$. 


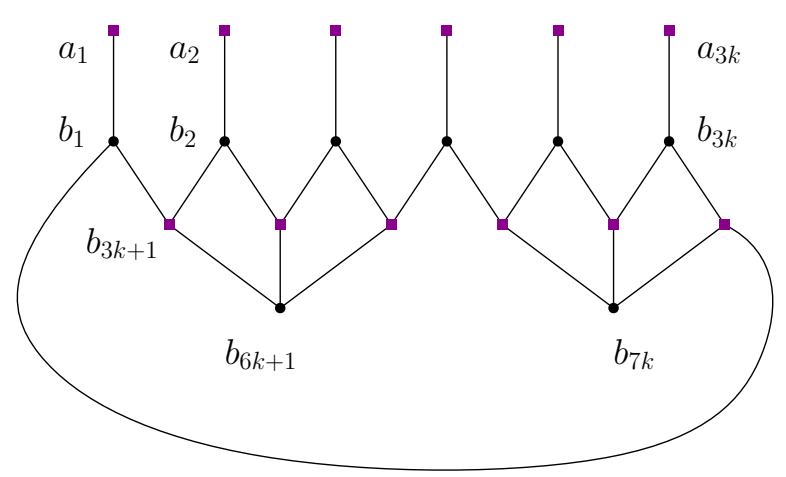

Figure 2: A subgraph of $G_{1}$ for $k=2$, depicting the changes in Step I.

\subsubsection{Step III}

At this step, we diverge from the work in [Ple84]. In Plesnik's hardness proof of Cubic Subgraph, the author substituted a complete bipartite graph at each $a_{i}$ so the resulting graph has a cubic subgraph if and only if it has the desired subgraph in Step I. We proceed by using a grid-like substitution at each $a_{i}$ into two copies of $G_{1}$.

Let $G_{2}$ be the graph obtained after the following substitution: At each vertex $a_{i}$, we substitute the following gadget.

For $j=1,2,3$, Let $S_{i, j} \in S$ be the 3 -element subsets containing $a_{i}$. Add vertices $u_{i, j}, w_{i, j}, c_{u_{i, j}}, c_{w_{i, j}}$ for $j=1,2,3$. For $j=1,2,3$, create the edges

$$
w_{i, j-1} u_{i, j}, u_{i, j} w_{i, j}, w_{i, j} S_{i, j}, u_{i, j} c_{u_{i, j}}, w_{i, j} c_{w_{i, j}}, c_{u_{i, j}} c_{w_{i, j}} .
$$

Here we understand $w_{i, 0}=b_{i}$.

Next, create a copy $G_{2}^{\prime}$ of $G_{2}$. For each $v \in G_{2}$, we let $v^{\prime}$ denote the corresponding copy in $G_{2}^{\prime}$. Also, add the edges $c_{w_{i, j}} c_{w_{i, j}}^{\prime}, c_{u_{i, j}} c_{u_{i, j}}^{\prime}$ for $1 \leq i \leq 3 k, 1 \leq j \leq 3$.

See Figure 3. The square and circular vertices depict a bipartition of $G$.

Define $O_{i, j}:=\left\{u_{i, j}, w_{i, j}, c_{u_{i, j}}, c_{w_{i, j}}\right\}$. Let us call the vertices $O_{i}:=\bigcup_{j=1}^{3} O_{i, j}$ the ore of $b_{i} \in B_{1}$ and similarly for $b_{j}^{\prime} \in B_{1}^{\prime}$.

Let this new graph be $G$. In summary we have the vertex sets $B:=B_{3} \cup B_{2} \cup B_{1}, S$ and $O_{i}, 1 \leq i \leq 3 k$ coming from the substitution in $G_{1}$. Similarly $B^{\prime}, S^{\prime}, O_{i}^{\prime}, 1 \leq i \leq 3 k$ are the vertex sets from $G_{1}^{\prime}$. Let $A=B \cup S \cup \bigcup_{1 \leq i \leq 3 k} O_{i}$ and $A^{\prime}=B^{\prime} \cup S^{\prime} \cup \bigcup_{1 \leq i \leq 3 k} O_{i}^{\prime}$ be the vertices which originated from $G_{1}, G_{1}^{\prime}$ respectively, so $V(G)=A \cup A^{\prime}$.

\subsubsection{Locally Regular Graphs}

Before we can prove correctness we need to introduce the concept of locally regular graphs which our proof will heavily rely on.

Let $G$ be a graph and $v \in V(G)$. Here we introduce a common definition $N(v):=\{w \in V(G): v w \in E(G)\}$

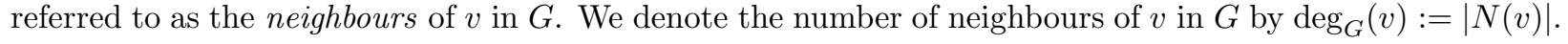

Definition 2.1 (Locally Regular) Let $G$ be an arbitrary graph and put $\varnothing \neq V^{\prime} \subseteq V$. Suppose $G$ contains a subgraph $H$ such that for all $v \in V^{\prime} \cap V(H), \operatorname{deg}_{H}(v)=r$ where $r \geq 1$ is a constant.

We say that $H$ is a locally $\left(V^{\prime}, r\right)$-regular subgraph of $G$. 


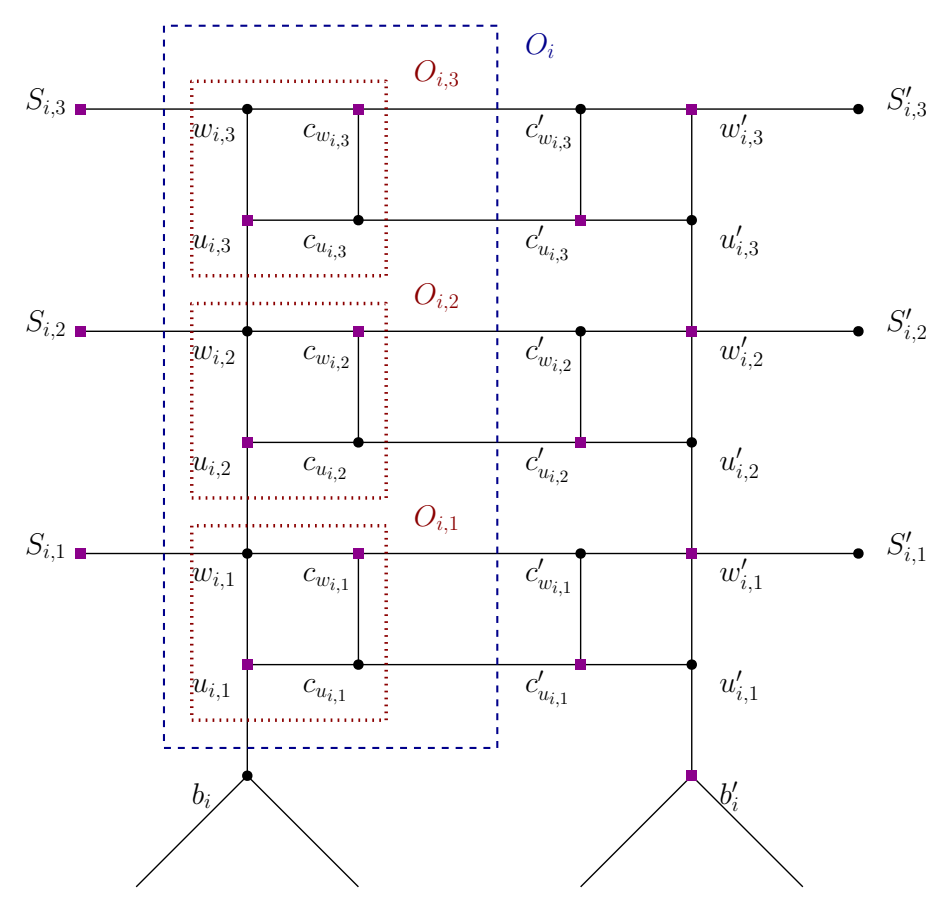

Figure 3: The substitution at what used to be $a_{i}, a_{i}^{\prime}$.

Next, Lemma 2.5 essentially shows the following: Given a locally $\left(V^{\prime}, r\right)$-regular subgraph $H$ of a graph $G$ where $G\left[V^{\prime}\right]$ is highly vertex-connected and there only a few vertices $v \in V^{\prime}$ for which $\operatorname{deg}_{G}(v) \neq r$, then $V(H)$ either contains all of $V^{\prime}$ or is disjoint from $V^{\prime}$

Before stating the lemma, let us introduce a notation. For any graph $G$ and $V^{\prime} \subseteq V(G)$, put $\Delta_{V^{\prime}}:=$ $\max _{v \in V^{\prime}} \operatorname{deg}_{G}(v)$.

Lemma 2.5 (Propagation) Let $G$ be an arbitrary graph and $\varnothing \neq V^{\prime} \subseteq V(G)$ be such that $G\left[V^{\prime}\right]$ is $\kappa$ vertex-connected for some $\kappa \geq 1$.

Let $H$ be a locally $\left(V^{\prime}, r\right)$-regular subgraph of $G$ for some $r \geq 1$.

Put $V_{r}:=\left\{v \in V^{\prime}: \operatorname{deg}_{G}(v)=r\right\}$. Then the following hold:

(i) If

$$
\kappa-\left|V^{\prime} \backslash V_{r}\right| \geq 1
$$

and $V_{r} \cap V(H) \neq \varnothing$, then $V^{\prime} \subseteq V(H)$.

(ii) If

$$
\kappa-\left|V^{\prime} \backslash V_{r}\right| \geq \max \left(\Delta_{V^{\prime}}-r, 1\right)
$$

then $V^{\prime} \nsubseteq V(H)$ implies $V^{\prime} \cap V(H)=\varnothing$.

See Figure 4. The green vertices reside in $V_{r}$ while the purple vertices do not. Notice how $P_{2}$ potentially leaves $H$ but $V\left(P_{1}\right) \subseteq V(H)$ as all internal vertices have degree $r=3$.

Proof (i): Let $G$ be an arbitrary graph and $\varnothing \neq V^{\prime} \subseteq V(G)$ be such that $G\left[V^{\prime}\right]$ is $\kappa$-vertex-connected for some $\kappa \geq 1$. Let $H$ be a locally $\left(V^{\prime}, r\right)$-regular subgraph of $G$ for some $r \geq 1$.

Suppose now that $V_{r} \cap V(H) \neq \varnothing$ and $\kappa-\left|V^{\prime} \backslash V_{r}\right| \geq 1$. Pick $v \in V_{r} \cap V(H)$ and fix any $v \neq w \in V^{\prime}$. The goal is to show that $w \in V(H)$. This implies $V^{\prime} \subseteq V(H)$ by the arbitrary choice of $w$. 


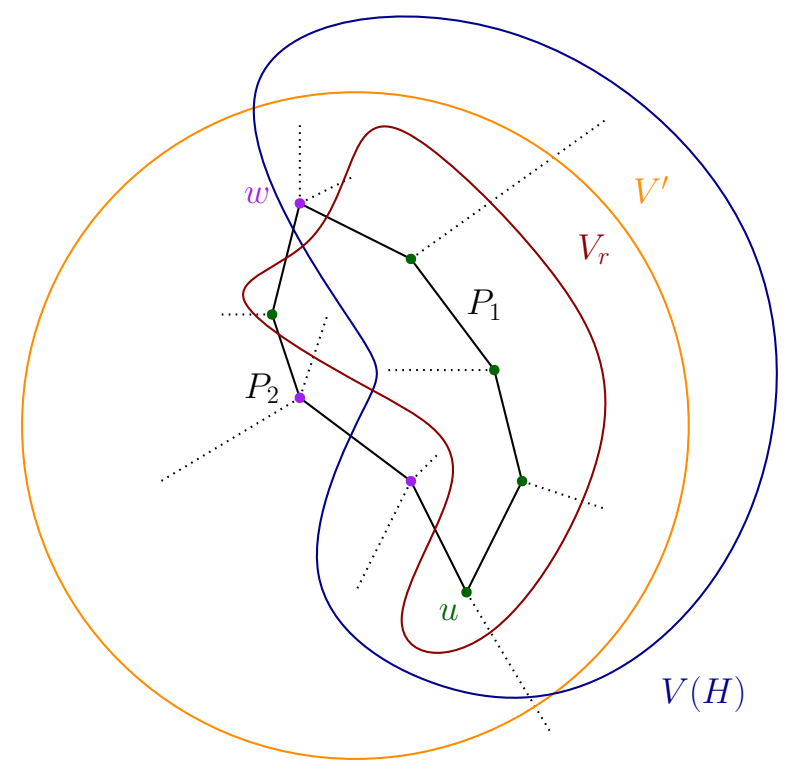

Figure 4: An illustration of Lemma 2.5 with $r=3, \kappa=2$ and $\Delta_{V^{\prime}}=4$.

Remark that $v, w \in V^{\prime}$. Hence by Menger's theorem, there are $\kappa$ internally vertex-disjoint $v w$-paths in $G\left[V^{\prime}\right]$ $P_{1}, P_{2}, \ldots P_{\kappa}$. If one of these paths contains no internal vertices, then $w \in N(v)$ and the definition of local regularity yields $w \in V(H)$. We proceed assuming all paths have internal vertices.

Hence, by the assumption that $\kappa-\left|V^{\prime} \backslash V_{r}\right| \geq 1$ and the pigeonhole principle, at least one of these paths, say $P_{1}$, is composed internally only of vertices from $V_{r}$. Write $P_{1}: u, v_{1}, v_{2}, \ldots, v_{\ell}, w$. Thus $\operatorname{deg}_{G}\left(v_{i}\right)=r$ for all $1 \leq i \leq \ell$.

Since $u \in V^{\prime} \cap V(H)$, we can apply the definition of local regularity to see that $v_{1} \in V(H)$. Repeat this argument to see that $v_{2}, \ldots, v_{\ell} \in V(H)$. Finally, since $w \in N\left(v_{\ell}\right)$, apply the definition of local regularity once more to conclude that $w \in V(H)$.

This concludes the proof by our initial remark.

Proof (ii): Suppose now that $\kappa-\left|V^{\prime} \backslash V_{r}\right| \geq \max \left(\Delta_{V^{\prime}}-r, 1\right)$ holds and there is some vertex $u \in V^{\prime} \backslash V(H)$. Let $u \neq w \in V^{\prime}$. There are again $\kappa$ internally vertex-disjoint $u w$-paths in $G\left[V^{\prime}\right]$, say $P_{1}, P_{2}, \ldots, P_{\kappa}$.

Case I: $w \in V_{r}$ Since $\kappa-\left|V^{\prime} \backslash V_{r}\right| \geq 1$, and $w \in V_{r} \cap V(H) \neq \varnothing$, an application of (i) yields $V^{\prime} \subseteq V(H)$. This contradicts the existence of $u$.

Case II: $w \in V^{\prime} \backslash V_{r}$ There are at most $\left|V^{\prime} \backslash V_{r}\right|-1$ vertices from $V^{\prime} \backslash V_{r}$ which can be internal vertices within our $\kappa$ paths. We will consider $u$ as an internal vertex. By the assumption that $\kappa-\left|V^{\prime} \backslash V_{r}\right| \geq \Delta_{V^{\prime}}-r$ and the pigeonhole principle, at least $\kappa-\left(\left|V^{\prime} \backslash V_{r}\right|-1\right) \geq \Delta_{V^{\prime}}-r+1$ paths are composed internally of vertices only from $V_{r}$.

Consider such a path $P_{i}: u, v_{1}, v_{2}, \ldots, v_{\ell}, w$ composed internally of vertices from $V_{r}$. Since $u \notin V(H), v_{1}$ cannot attain degree $r$ in $H$ thus $v_{1} \notin V(H)$ as well. Repeating this argument, we see that $v_{2}, \ldots, v_{\ell} \notin V(H)$.

Since there are at least $\Delta_{V^{\prime}}-r+1$ such paths, $\operatorname{deg}_{H}(w) \leq \Delta_{V^{\prime}}-\left(\Delta_{V^{\prime}}-r+1\right)=r-1$. Thus the definition of local regularity asserts that $w \notin V(H)$.

It follows that $V^{\prime} \cap V(H)=\varnothing$ by the arbitrary choice of $w$ as desired. 


\subsubsection{Correctness}

Now we will show that $G$ contains a two from cubic subgraph if and only if there is an exact cover of $X$ by 3 -sets.

Definition 2.2 (Trivial Two from Cubic Subgraph) Let $H$ be a two from cubic subgraph of $G$ with special vertices $u, v$. If $u v \in E(G) \backslash E(H)$, then $H+u v$ is a cubic subgraph of $G$ and $H$ is a trivial two from cubic subgraph of $G$.

Observe that $G$ has a cubic subgraph if and only if it has a trivial two from cubic subgraph.

In Theorem 2.10 we show that an exact cover exists if and only if $G$ contains a (trivial two from) cubic subgraph. This proves the forward direction as the existence of an exact cover implies the existence of a two from cubic subgraph. The converse is also simplified, since if there is a trivial two from cubic subgraph, it implies the existence of an exact cover by 3 -sets. We need only prove that if $G$ contains a non-trivial two from cubic subgraph, then there is an exact cover. This is done in Proposition 2.11.

First, we prove that an instance of EXACT COVER BY 3-SETS is a "yes" instance if and only if the constructed gadget graph contains a (trivial two from) cubic subgraph.

For graphs $H, H^{\prime}$, we say $H^{\prime}$ was obtained from $H$ by adding a path if $H^{\prime}$ contains a path $P$ with endpoints $u \neq v$ such that $V(H)=V\left(H^{\prime}\right) \backslash(V(P) \backslash\{u, v\})$ and $E(H)=E\left(H^{\prime}\right) \backslash E(P)$.

We will use the following elementary graph theory result.

Proposition 2.6 (Ear Decomposition; [Die16]) A graph is 2-vertex-connected if and only if it can be constructed from a cycle by successively adding a path.

Lemma 2.7 $G[B]$ and $G\left[O_{i, j}\right]$ for all $i, j$ are 2-vertex-connected.

Proof: $G[B]$ is 2-Vertex-Connected: Let us argue by ear decomposition (Proposition 2.6). Notice first that $G\left[B_{1} \cup \overline{\left.B_{2}\right]}\right.$ is a cycle. We successively add each vertex $b_{6 k+j} \in B_{3}$ and its incident edges to obtain $G[B]$ as follows: First add the path consisting of vertices $b_{3 k+3 j-2}, b_{6 k+j}, b_{3 k+3 j-1}$. Then add the path consisting of vertices $b_{6 k+j}, b_{3 k+3 j}$.

Thus $G[B]$ is indeed 2-vertex-connected.

$G\left[O_{i, j}\right]$ is 2-Vertex-Connected: $G\left[O_{i, j}\right]$ is simply a cycle and hence 2-vertex-connected.

Lemma 2.8 Suppose $H$ is a locally $(B, 3)$-regular subgraph of $G$. Let $v \in B$ be arbitrary.

Then $v \in V(H)$ implies $B \subseteq V(H)$ and $v \notin V(H)$ implies $B \cap V(H)=\varnothing$. In particular, either $B \subseteq V(H)$ or $B \cap V(H)=\varnothing$.

Proof: Since $G[B]$ is 2-vertex-connected by Lemma 2.7, we can simply apply Lemma 2.5 with $V^{\prime}=B, \kappa=$ $2, r=3, \Delta_{B}=3$.

Note that $V^{\prime} \backslash V_{r}=\varnothing$. Hence both assumptions from Lemma 2.5 (Equation (9), Equation (10)) are satisfied.

Lemma 2.9 Suppose $H$ is a locally $\left(O_{i, j}, 3\right)$-regular subgraph of $G$ for some $i, j$ and fix some $v \in O_{i, j}$.

Then $v \in H$ implies $O_{i, j} \subseteq V(H)$ and $v \notin H$ implies $O_{i, j} \cap V(H)=\varnothing$. In particular, either $O_{i, j} \subseteq V(H)$ or $O_{i, j} \cap V(H)=\varnothing$.

Proof: Since $G\left[O_{i, j}\right]$ is 2-vertex-connected by Lemma 2.7, we can simply apply Lemma 2.5 with $V^{\prime}=$ $O_{i, j}, \kappa=2, r=3, \Delta_{O_{i, j}}=4$.

Observe that $\left|V^{\prime} \backslash V_{r}\right| \leq 1$. Hence both assumptions from Lemma 2.5 (Equation (9), Equation (10)) are satisfied.

Theorem 2.10 There is an exact cover of $X$ by 3-sets if and only if $G$ contains a cubic subgraph. 
Proof: $(\Longrightarrow)$ Let $Y \subseteq S$ be the exact cover of $X$.

We will describe the vertices from $A$ of a cubic subgraph and take the exact copy within $A^{\prime}$.

First, take all vertices $b \in B$. Fix $b_{i} \in B_{1}$. There is exactly one $S_{i, j} \in Y$ such that $a_{i} \in S_{i, j}$. Let $P_{i}$ be the unique $b_{i} S_{i, j}$-path in the ore of $b_{i}$. Put $Q_{i}:=\bigcup_{j: O_{i, j} \cap V\left(P_{i}\right) \neq \varnothing} O_{i, j}$. Then we take $B \cup\left(\bigcup_{i} Q_{i}\right) \cup Y$ as well as their copies in $A^{\prime}$ to be the vertex set of our cubic subgraph.

$(\Longleftarrow)$ Let $H$ be a cubic subgraph of $G$. First, we claim that $B \subseteq V(H)$.

Suppose otherwise. By Lemma 2.8, which states that if $H$ is locally $(B, 3)$-regular then either $B \subseteq V(H)$ or $B \cap V(H)=\varnothing$, we see that $B \cap V(H)=\varnothing$.

Fix some $u_{i, 1}$. Lemma 2.7 states that $G\left[O_{i, 1}\right]$ is 2-vertex-connected. From our work above, we must have $u_{i, 1} \notin V(H)$. Lemma 2.9 states that if $H$ is locally $\left(O_{i, j}, 3\right)$-regular then either $O_{i, j} \subseteq V(H)$ or $O_{i, j} \cap V(H)=$ $\varnothing$. Since the former cannot happen, $O_{i, 1} \cap V(H)=\varnothing$. Thus $u_{i, 2} \notin V(H)$.

Repeating this argument for $u_{i, 2}, u_{i, 3}$ shows that $V(H) \subseteq S$. But then $H$ cannot be 3-regular. By contradiction, $B \subseteq V(H)$.

Finally, let us fix some $b_{i} \in B_{1}$ and remark that $b_{i} \in V(H)$ implies $u_{i, 1} \in V(H)$. Once more, apply Lemma 2.9 to see that $O_{i, 1} \subseteq V(H)$. If $H$ contains the edge $w_{i, 1} S_{i, 1}$, then it contains no more vertices in the ore of $b_{i}$. Otherwise, we repeat this argument to see that $H$ also contains $O_{i, 2}$, etc. All in all, there is exactly one subset $S_{j} \in S$ which is adjacent to the ore of $b_{i}$.

Put $Y:=S \cap V(H)$ as the sub-collection. By our work above, $Y$ covers each element exactly once. Thus $Y$ is the desired exact cover.

Now, we prove the converse: If $G$ contains a two from cubic subgraph, then there is an exact cover of $X$ by 3 sets. The key insight is that the structure of our gadget forces the two inconvenient vertices to be in different copies. Then, we apply Lemma 2.5 multiple times to show that the arguments made in Theorem 2.10 still follow despite the existence of those two vertices.

Definition 2.3 (Lacking) Let $H$ be a non-trivial two from cubic subgraph. We say $v \in V(H)$ is lacking if $\operatorname{deg}_{H}(v)=2$.

Notice that if $v$ is lacking with $v w \in E(G) \backslash E(H)$ and $\operatorname{deg}_{G}(w)=3$, then it must be that $w \notin V(H)$ or else $H$ was not a non-trivial two from cubic subgraph.

Proposition 2.11 If $G$ contains a two from cubic subgraph, then there is an exact cover of $X$ by 3-sets.

Recall that $A=B \cup S \cup \bigcup_{1 \leq i \leq 3 k} O_{i}$ and $A^{\prime}=B^{\prime} \cup S^{\prime} \cup \bigcup_{1 \leq i \leq 3 k} O_{i}^{\prime}$ are the vertices which originated from $G_{1}, G_{1}^{\prime}$ respectively, so $V(G)=A \cup \dot{\cup} A^{\prime}$.

Proof: Let $H$ be a non-trivial two from cubic subgraph.

If $A \cap V(H) \neq \varnothing$ and no vertex of $A$ is lacking, then there is an exact cover. We prove this in Lemma 2.12. Thus it suffices to consider the cases where $A \cap V(H)=\varnothing$ or some vertex of $A$ is lacking.

We claim that $A \cap V(H) \neq \varnothing \neq A^{\prime} \cap V(H)$. We prove this claim in Lemma 2.13. Moreover, if either $A, A^{\prime}$ does not contain a lacking vertex, then there is an exact cover. We defer the proof to Lemma 2.14. Thus we may as well assume that each of $A, A^{\prime}$ contains a lacking vertex or we are done. It is also well-defined to say the lacking vertex of $A$ or $A^{\prime}$.

We will show in Lemma 2.16 and Lemma 2.17 respectively that $B \subseteq V(H)$ and $S$ does not contain a lacking vertex. Thus with the lacking vertex of $A$ not in either $B, S$. It must thus reside in the ore of some $b_{i}$.

For all other ores of $b_{j}, j \neq i$, Lemma 2.9 forces that ore to be adjacent to exactly one vertex of $S$. But we must have $|\delta(S \cap V(H))| \equiv 0 \bmod 3$. The only way for this to hold is if the ore of $b_{i}$ is also adjacent to exactly one vertex of $S$. 
Thus despite the ore of $b_{i}$ containing a lacking vertex, $Y:=S \cap V(H)$ covers every ground set element exactly once, concluding our proof.

Lemma 2.12 Let $H$ be a non-trivial two from cubic subgraph. Suppose $A \cap V(H) \neq \varnothing$ and that no vertex of $A$ is lacking.

Then there is an exact cover.

Proof: The proof of this claim is similar to the reverse direction for Theorem 2.10.

First note that $A \cap V(H) \neq \varnothing$ implies that $B \subseteq V(H)$. Indeed, if any vertex $b \in B$ is not a vertex of $V(H)$, then the Lemma 2.8 ensures that $B \cap V(H)=\varnothing$. But then repeated applications of Lemma 2.9 show that $H$ does not contain any ore vertices. So $H$ contains no vertices of $A$, which is a contradiction.

Since $B \subseteq V(H)$, let us focus on the ores. For every ore of some $b_{i}$, repeated application of Lemma 2.9 force $H$ to choose exactly one edge of the form $w_{i, j} S_{i, j}$.

Thus taking $Y:=V(H) \cap S$ defines an exact cover of $X$.

Lemma 2.13 Let $H$ be a non-trivial two from cubic subgraph.

Then $A \cap V(H) \neq \varnothing \neq A^{\prime} \cap V(H)$.

Proof: By symmetry, it suffices to consider the case where $A^{\prime} \cap V(H)=\varnothing$.

Observe that $\left|B_{1} \cap V(H)\right| \leq 2$ since if the ore of $b_{i} \in B_{1}$ does not contain a lacking vertex, then $c_{w_{i, j}} \notin V(H)$ for all $j=1,2,3$. Hence by Lemma 2.9, $O_{i, j} \cap V(H)=\varnothing$ for each $j=1,2,3$. The claim then follows.

But then $\left|B_{2} \cap V(H)\right| \leq 1$ since if $b_{j} \in B_{2}$ is not adjacent to both its neighbors in $B_{1}$, it cannot attain degree 3. This implies $B_{3} \cap V(H)=\varnothing$ as no $b_{i} \in B_{3}$ attains degree 3. These restrictions show that in fact $B \cap V(H)=\varnothing$.

If $H$ does not contain any ore vertices, it is the empty graph.

Suppose $H$ partially contains exactly one ore of $b_{i} \in B_{1}$. Then at least one vertex of the ore must be lacking. But then it is not possible for a vertex of $S$ to be of degree 2 or 3 so $S \cap V(H)=\varnothing$. Specifically, $w_{i, 3} \notin V(H)$. By an inductive argument, it is not hard to see that $O_{i, 3}, O_{i, 2}, O_{i, 1} \cap V(H)=\varnothing$. But then $H$ is actually the empty graph.

Finally, suppose $H$ partially contains exactly two ores of $b_{i} \neq b_{j} \in B_{1}$. Observe that it can partially contain at most two ores in total, as each of them consumes one lacking vertex. But then $H$ cannot contain any vertex of $S$ since any vertex of $S$ cannot achieve degree 3. Similar to the previous case, $H$ is actually the empty graph.

Lemma 2.14 Let $H$ be a non-trivial two from cubic subgraph.

If either $A, A^{\prime}$ does not contain a lacking vertex, there is an exact cover of $X$ by 3-sets.

Proof: Suppose not. By Lemma 2.13, each of $A, A^{\prime}$ is not empty and one of them, say $A$, does not contain a lacking vertex.

The result follows then from Lemma 2.12.

Lemma 2.15 Let $H$ be a non-trivial two from cubic subgraph where both $A, A^{\prime}$ contains a lacking vertex.

Either $B \subseteq V(H)$ or $B \cap V(H)=\varnothing$.

Proof: By Lemma 2.7, $G[B]$ is 2-vertex-connected.

Suppose now that the statement does not hold. There is a vertex $b \in B \cap V(H)$ and another vertex $b^{*} \in B \backslash V(H)$.

By 2-vertex-connectedness, there are two vertex disjoint $b b^{*}$-paths $P_{1}, P_{2}$ in $G[B]$. Since $b^{*} \notin V(H)$, there are two distinct edges $b_{i} b_{j}, b_{k} b_{\ell}$ which are contained in $P_{1}, P_{2}$ respectively such that $b_{i}, b_{k} \in V(H), b_{j}, b_{\ell} \notin V(H)$. 
If $b=b_{i}=b_{k}$, then $\operatorname{deg}_{H}(b)=1$ which is a contradiction. Otherwise, $b_{i} \neq b_{k}$ and we notice that $\operatorname{deg}_{H}\left(b_{i}\right), \operatorname{deg}_{H}\left(b_{k}\right) \leq 2$. But only one of the two can be lacking and we have a contradiction.

Lemma 2.16 Let $H$ be a non-trivial two from cubic subgraph where both $A, A^{\prime}$ contains a lacking vertex.

$B \subseteq V(H)$ and does not contain a lacking vertex.

Proof: By Lemma 2.15, either $B \subseteq V(H)$ or $B \cap V(H)=\varnothing$.

$B \subseteq V(H)$ : Suppose $B \cap V(H)=\varnothing$. Repeated application of Lemma 2.9 to each ore shows that if some ore

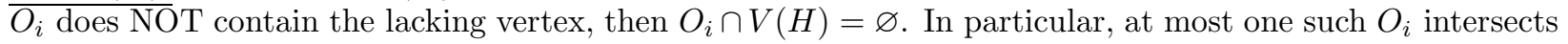
non-trivially with $V(H)$.

But then $S \cap V(H)=\varnothing$ as no vertex of $S$ can attain degree 3 within $H$. It follows that $w_{i, 3} \notin V(H)$. A similar argument to the proof of Lemma 2.13 shows that $O_{i} \cap V(H)=\varnothing$ as well.

This is a contradiction since we assumed that $V(H) \cap A \neq \varnothing$. Thus $B \subseteq V(H)$. We now show that no vertex of $B$ is lacking.

$B$ contains no lacking vertices: This is easy for $b \in B_{3} \cup B_{2}$. Indeed, any such lacking vertex implies one of its neighbors in $B$ is not in $H$. This would contradict the assumption that $B \subseteq V(H)$. Similarly, if $b \in B_{1}$ is not adjacent to one of its neighbors in $B$, then we cannot have $B \subseteq V(H)$. The only possibility is some $b_{i} \in B_{1}$ not adjacent to $u_{i, 1}$.

But then by the repeated application of Lemma 2.9, we see that $H$ does not contain any vertices from the ore of $b_{i}$. But Lemma 2.9 forces $H$ to contain vertices from all other ores. Moreover, each such ore is adjacent to exactly one vertex in $S$. Thus $|\delta(S \cap V(H))| \equiv-1 \bmod 3$ which is a contradiction as no vertex of $S$ is lacking.

Lemma 2.17 Let $H$ be a non-trivial two from cubic subgraph where both $A, A^{\prime}$ contains a lacking vertex.

$S$ does not contain a lacking vertex.

Proof: Suppose otherwise, that some vertex of $S$ is lacking.

By Lemma 2.16, $B \subseteq V(H)$. Similar to before, repeated application of Lemma 2.9 shows that $A$ contains vertices from each ore, and every ore is adjacent to exactly one vertex in $S$.

It follows that $|S \cap V(H)| \equiv 0$ mod 3. But since $S$ contains a lacking vertex, the above is impossible.

In all cases, whether $H$ is a trivial or non-trivial two from cubic subgraph, there is an exact cover. Moreover, the degree bound from Theorem 2.1 holds since $G$ is bipartite with maximum degree 4.

\section{Positive Results}

In the case of $b \leq 2$, we explore several variants of $b$-matching games for which the nucleolus can be efficiently computed.

First, we will state an important ingredient.

Let $\Gamma=(N, \nu)$ be a cooperative game. For $\mathscr{S} \subseteq \mathcal{S}$ and $x \in \mathbb{R}^{N}$, write $\theta^{\mathscr{S}}(x) \in \mathbb{R}^{\mathscr{S}}$ to denote the restricted vector containing the excess values $e(S, x)$ for $S \in \mathscr{S}$ in non-decreasing order of excess.

Definition 3.1 (Characterization Set) Let $\mathscr{S} \subseteq \mathcal{S}$ be a subset of the non-trivial coalitions.

We say $\mathscr{S}$ is a characterization set for the nucleolus of the cooperative game $\Gamma=(N, \nu)$ if the lexicographic maximizer of $\theta^{\mathscr{S}}(x)$ is a singleton that lexicographically maximizes the unrestricted vector $\theta(x)$.

Intuitively, for $S \in \mathcal{S} \backslash \mathscr{S}$, we can drop the constraint corresponding to $S$ from the Kopelowitz Scheme when computing the nucleolus. 
Proposition 3.1 Let $\Gamma=(N, \nu)$ be a cooperative game with non-empty core. Suppose $\mathscr{S}$ is a polynomial sized characterization set for the nucleolus of $\Gamma$.

The nucleolus of $\Gamma$ is polynomial time computable.

Let $\mathscr{S} \subseteq \mathcal{S}$ be a characterization set of the nucleolus of some game $\Gamma$. Consider the following tweak of the $\ell$-th iteration of Kopelowitz Scheme $\left(P_{\ell}^{\prime}\right)$ (with optimal value $\epsilon_{\ell}^{\prime}$ ) where we only have constraints corresponding to coalitions in the characterization set $\mathscr{S}$ instead of every coalition. The sets $\mathcal{S}_{\ell}$ are defined in symmetric fashion as the coalitions from $\mathscr{S}$ which are fixed in $\left(P_{\ell}\right)$ but not at any prior $\left(P_{i}\right)$.

$$
\begin{array}{cl}
\max \epsilon & \left(P_{\ell}^{\prime}\right) \\
x(S)=\nu(S)-\epsilon_{i}^{\prime} & \forall 0 \leq i<\ell, \forall S \in \mathcal{S}_{i} \\
x(S)-\nu(S) \geq \epsilon & \forall S \in \mathscr{S} \backslash \bigcup_{i=0}^{\ell-1} \mathcal{S}_{i}
\end{array}
$$

Proof: The tweaked Kopelowitz Scheme computes the lexicographic maximizer of $\theta^{\mathscr{S}}$. Since $\mathscr{S}$ is polynomially sized, each linear program in the scheme can be solved in polynomial time.

We are now ready to state the theorem found in [GGZ98].

Theorem 3.2 ([GGZ98]) Let $\Gamma=(N, \nu)$ be a cooperative game with non-empty core.

The non-empty collection $\mathscr{S} \subseteq \mathcal{S}$ is a characterization set for the nucleolus of $\Gamma$ if for every $S \in \mathcal{S} \backslash \mathscr{S}$ there exists a non-empty subcollection $\mathscr{S}_{S}$ of $\mathscr{S}$ such that

(i) For all $T \in \mathscr{S}_{S}$ and core allocations $x, e(T, x) \leq e(S, x)$.

(ii) There are scalars $\lambda_{T} \in \mathbb{R}$ such that the characteristic vector $\chi_{S} \in\{0,1\}^{N}$ of $S$ satisfies $\chi_{S}=$ $\sum_{T \in \mathscr{S}_{S} \cup\{N\}} \lambda_{T} \chi_{T}$.

Corollary 3.2.1 Let $\Gamma=(N, \nu)$ be a not necessarily simple weighted b-matching game with non-empty core. Define

$$
\mathscr{S}:=\{S \in \mathcal{S}: \text { For all maximum weight b-matchings } M \text { of } G[S], G[S][M] \text { is connected }\} .
$$

Then $\mathscr{S}$ is a characterization set for the nucleolus of $\Gamma$.

Proof: Fix $S \in \mathcal{S} \backslash \mathscr{S}$. Let $M$ be such that $G[S][M]$ has the maximal number of connected components among all maximum weight $b$-matchings of $G[S]$. By the choice of $S, G[S][M]$ must be disconnected. Let $T_{1}, T_{2}, \ldots, T_{k}$ be the components of $G[S][M]$ for some $k \geq 2$. We claim that each $T_{i} \in \mathscr{S}$. Suppose otherwise, that there is some $T_{i} \notin \mathscr{S}$. Then there is a maximum weight $b$-matching $M_{i}$ in $G\left[T_{i}\right]$ which is disconnected. But then $M \backslash E\left(T_{i}\right) \cup M_{i}$ is a maximum weight $b$-matching in $G[S]$ with strictly more connected components, a contradiction.

Suppose $x$ is a core allocation. Since $x(S)=\sum_{i=1}^{k} x\left(T_{i}\right)$ and $\nu(S)=\sum_{i=1}^{k} \nu\left(T_{i}\right)$, we have $\sum_{i=1}^{k} e\left(S_{i}, x\right)=$ $e(S, x)$. In particular, condition (ii) of Theorem 3.2 is satisfied. But all excesses are non-negative as $x$ is a core allocation, hence each $e\left(S_{i}, x\right) \leq e(S, x)$ and condition (i) of Theorem 3.2 is also satisfied.

The result follows by Theorem 3.2 .

Lemma 3.3 Let $\Gamma=(N, \nu)$ be a not necessarily simple weighted b-matching game with non-empty core. Suppose

$$
\mathscr{S}:=\{S \in \mathcal{S}: \text { For all maximum weight b-matchings } M \text { of } G[S], G[S][M] \text { is connected }\} .
$$

is polynomially sized. 
Then the nucleolus of $\Gamma$ is polynomial time computable.

Proof: Apply Proposition 3.1 and Corollary 3.2.1.

\subsection{Simple b-Matching Games}

We now present a proof for the first claim of Theorem 1.2, which is stated again below for convenience. Let $G$ be a simple bipartite graph with bipartition $N=A \dot{\cup} B$ and $k \geq 0$ a universal constant independent of $|N|$. Let $b \leq 2$ be some node-incidence capacity.

(i) Suppose $b_{v}=2$ for all $v \in A$ but $b_{v}=2$ for at most $k$ vertices of $B$, then the nucleolus of the simple weighted $b$-matching game on $G$ can be computed in polynomial time.

(ii) If $b \equiv 2$, then the nucleolus of the non-simple weighted $b$-matching game on $G$ can be computed in polynomial time.

Proof (Theorem 1.2(i)): By Lemma 3.3, it suffices to show that any component of a $b$-matching in some arbitrary induced subgraph $G[S]$ has at most $2 k+3$ vertices. If we show this, then the set

$$
\mathscr{S}:=\{S \in \mathcal{S}: \text { For all maximum weight } b \text {-matchings } M \text { of } G[S], G[S][M] \text { is connected }\}
$$

is polynomially sized since it is contained in the subsets of $V(G)$ of size at most $2 k+3$.

Let $C$ be a component of $G[S][M]$ for some $S \subseteq N$ and maximum weight $b$-matching $M$ of $G[S]$.

If $C$ is a cycle, then exactly half the vertices of $C$ are from $B$ with $b_{v}=2$. It follows that $|C| \leq 2 k$.

Suppose now that $C$ is some path. By deleting at both endpoints and one more vertex, we may assume that every other vertex in the path are from $B$ with $b_{v}=2$. Thus $|C| \leq 2 k+3$ as required.

\subsection{Non-Simple 2-Matching Games}

In the case where we allow for edges to be included multiple times in a 2-matching, we leverage core nonemptiness and the non-existence of odd cycles to compute the nucleolus in polynomial time.

Lemma 3.4 Let $G$ be an arbitrary graph with edge weights $w: E \rightarrow \mathbb{R}$.

The core of the weighted non-simple 2-matching game on $G$ is non-empty.

Consider the following LP formulation of the maximum weight non-simple $b$-matching from [Sch03].

$$
\begin{aligned}
& \max w^{T} y & & (P) \\
y(\delta(v)) & \leq b_{v} & & \forall v \in V \\
y(E[U]) & \leq\left\lfloor\frac{1}{2} b(U)\right\rfloor & & \forall U \subseteq V, b(U) \text { is odd } \\
y & \geq 0 & &
\end{aligned}
$$

Observe that for the case $b \equiv 2, b(U)$ is never odd and hence there are no constraints of the form Equation (16).

Thus the dual LP of non-simple 2-matching games can be simplified to the following.

$$
\begin{array}{rlrl}
\min 2^{T} x & & (D) \\
x_{u}+x_{v} & \geq w(u v) & & \forall u v \in E \\
x & \geq 0 & &
\end{array}
$$


Proof: Let $\bar{x}$ be an optimal solution to $(D)$. Since $2 \bar{x}(N)=\nu(N)$ by the integrality of $(P), \bar{x}$ is an allocation.

Fix a coalition $\varnothing \neq S \subsetneq N$. Define $\left(D_{S}\right)$ as the dual to the non-simple 2-matching LP (Equation (14)) on $G[S]$. Write $\left.\bar{x}\right|_{S}$ as the restriction of $\bar{x}$ to entries indexed by vertices of $S$.

Observe that $\left.\bar{x}\right|_{S}$ is feasible in $\left(D_{S}\right)$, thus $\nu(S) \leq \bar{x}(S)=\left.\bar{x}\right|_{S}(S)$ by weak duality and $\bar{x}(S)-\nu(S) \geq 0$.

By the arbitrary choice of $S, \bar{x}$ is a core allocation and consequently, the core is non-empty.

Notice that since $b \equiv 2$, we did not need to assume $G$ to be bipartite.

Lemma 3.5 For any bipartite graph, there is a maximum weighted non-simple 2-matching consisting only of parallel edges.

Proof: Let $M$ be a maximum weight non-simple 2-matching in $G$. Observe that the components of $G[M]$ are parallel edges, even cycles, and paths. Moreover, any path contains at least 2 edges, or else adding that single edge a second time to our matching can only increase the weight of the matching.

Let $x$ be the characteristic vector of $M$. We argue that all vertex solutions to Equation (14) contain no even cycles nor paths containing at least 2 edges. Hence an optimal vertex solution has the desired properties.

Case I: Even Cycles Suppose $C \subseteq M$ for some even cycle $C$. Enumerate the edges $C: e_{1}, e_{2}, e_{3}, \ldots, e_{k}$ for some $k \equiv 0 \bmod 2$.

For $i=0,1$, put $M^{(i)}:=M \backslash C \cup\left\{e_{j}, e_{j}: j \equiv i \bmod 2\right\}$. That is, double up every other edge of $C$.

Set $x^{(i)}$ to be the characteristic vector of $M^{(i)}$. Then $x=\frac{1}{2} x^{(0)}+\frac{1}{2} x^{(1)}$ and so $x$ was not a vertex solution.

Case II: Paths of Length At Least 2 Let $P \subseteq M$ be a path of length at least 2. Enumerate the edges

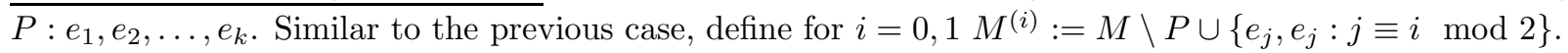

Set $x^{(i)}$ to be the characteristic vector of $M^{(i)}$. Then $x=\frac{1}{2} x^{(0)}+\frac{1}{2} x^{(1)}$ and so $x$ is not a vertex solution.

We are now ready to prove the second claim of Theorem 1.2.

Proof (Theorem 1.2(ii)): By Lemma 3.3, it suffices to show that if $|S| \geq 3$, then there is a 2-matching in $G[S]$ with multiple components.

But this is precisely what we proved in Lemma 3.5, concluding the proof.

Unfortunately, Lemma 3.5 does not hold when the graph is non-bipartite, even when we restrict ourselves to uniform edge weights. Indeed, consider the simple triangle. The maximum cardinality non-simple 2matching has size 3 . However, when we restrict ourselves to matchings composed of only parallel edges, the maximum matching we can obtain has cardinality 2 .

Similarly, Lemma 3.5 does not in general hold when there are some vertices $v$ where $b_{v}=1$. Consider the path of 3 edges where the endpoints have $b_{v}=1$ while the internal vertices have $b_{v}=2$. The maximum cardinality non-simple 2-matching has size 3 . However, if we only allow parallel edges, the maximum matching we can obtain again has cardinality 2 .

On the hopeful side, we note that a technique used to prove that the nucleolus of 1-matching games on general graphs with non-empty core can be computed in polynomial time [BKP12] could possibly extend to non-simple 2-matching games on general graphs. There, the authors demonstrated a bijection between the so-called maximum weight half-matchings of a graph and the maximum weight matchings of a gadget graph.

\section{Conclusion}

We showed that computing the nucleolus for simple bipartite $b$-matching games when $b \geq 3$ is $\mathcal{N} \mathcal{P}$-hard. When $b \leq 2$, we described a polynomial time algorithm for nucleolus computation in simple $b$-matching 
games on bipartite graphs, given that one side of the bipartition has a constant number of vertices $v$ where $b_{v}=2$. We also provided a polynomial time algorithm for computing the nucleolus of non-simple 2-matching games within bipartite graphs. This is a relaxation of the simple $b$-matching nucleolus problem when $b \equiv 2$. Our second positive result relies heavily on bipartiteness and the choice of $b \equiv 2$.

As mentioned, a next step following our work could be to examine the nucleolus computation algorithm for general graphs with non-empty core [BKP12] and determine whether it can be extended to non-simple 2-matchings in general graphs. Another future direction could be to provide an efficient algorithm for simple $b$-matching nucleolus computation in bipartite, and then in general graphs when $b \leq 2$ or prove that this is $\mathcal{N P}$-hard. As the LP-based schemes of Kopelowitz and Maschler have been the basis to our efforts, it may be of interest to explore combinatorial algorithms to compute the nucleolus. For instance, Hardwick gives a combinatorial algorithm for some special cases of the 1-matching game [Har17] and it would be interesting to give a combinatorial algorithm for the general case.

\section{References}

[AM85] Robert J Aumann and Michael Maschler. "Game theoretic analysis of a bankruptcy problem from the Talmud". In: Journal of economic theory 36.2 (1985), pp. 195-213.

[Bat+10] Mohammad Hossein Bateni, Mohammad Taghi Hajiaghayi, Nicole Immorlica, and Hamid Mahini. "The cooperative game theory foundations of network bargaining games". In: International Colloquium on Automata, Languages, and Programming. Springer. 2010, pp. 67-78.

[Bir+18] Péter Biró, Walter Kern, Daniël Paulusma, and Péter Wojuteczky. "The stable fixtures problem with payments". In: Games and economic behavior 108 (2018), pp. 245-268.

[Bir+19] Péter Biró, Walter Kern, Dömötör Pálvölgyi, and Daniel Paulusma. "Generalized Matching Games for International Kidney Exchange". In: Proceedings of the 18th International Conference on Autonomous Agents and MultiAgent Systems. 2019, pp. 413-421.

[BKP12] Péter Biró, Walter Kern, and Daniël Paulusma. "Computing solutions for matching games". In: International journal of game theory 41.1 (2012), pp. 75-90.

[BST05] Rodica Brânzei, Tamás Solymosi, and Stef Tijs. "Strongly essential coalitions and the nucleolus of peer group games". In: International Journal of Game Theory 33.3 (2005), pp. 447-460.

[CY92] Karen S Cook and Toshio Yamagishi. "Power in exchange networks: A power-dependence formulation". In: Social networks 14.3-4 (1992), pp. 245-265.

[DF08] Xiaotie Deng and Qizhi Fang. "Algorithmic cooperative game theory". In: Pareto Optimality, Game Theory And Equilibria. Springer, 2008, pp. 159-185.

[DFS09] Xiaotie Deng, Qizhi Fang, and Xiaoxun Sun. "Finding nucleolus of flow game". In: Journal of combinatorial optimization 18.1 (2009), pp. 64-86.

[Die16] Reinhard Diestel. Graph Theory. 5th. Vol. 173. GTM. 2016.

[DIN99] Xiaotie Deng, Toshihide Ibaraki, and Hiroshi Nagamochi. "Algorithmic aspects of the core of combinatorial optimization games". In: Mathematics of Operations Research 24.3 (1999), pp. 751766.

[DK10] Julia Drechsel and Alf Kimms. "Computing core allocations in cooperative games with an application to cooperative procurement". In: International Journal of Production Economics 128.1 (2010), pp. 310-321.

[DM65] Morton Davis and Michael Maschler. "The kernel of a cooperative game". In: Naval Research Logistics Quarterly 12.3 (1965), pp. 223-259.

[EK +12$]$ David Easley, Jon Kleinberg, et al. "Networks, crowds, and markets: Reasoning about a highly connected world". In: Significance 9.1 (2012), pp. 43-44. 
[Elk+07] Edith Elkind, Leslie Ann Goldberg, Paul Goldberg, and Michael Wooldridge. "Computational complexity of weighted threshold games". In: AAAI. 2007, pp. 718-723.

[Elk+09] Edith Elkind, Leslie Ann Goldberg, Paul W Goldberg, and Michael Wooldridge. "On the computational complexity of weighted voting games". In: Annals of Mathematics and Artificial Intelligence 56.2 (2009), pp. 109-131.

[FKK01] Ulrich Faigle, Walter Kern, and Jeroen Kuipers. "On the computation of the nucleolus of a cooperative game". In: International Journal of Game Theory 30.1 (2001), pp. 79-98.

[FKK06] Ulrich Faigle, Walter Kern, and Jeroen Kuipers. "Computing an element in the lexicographic kernel of a game". In: Mathematical methods of operations research 63.3 (2006), pp. 427-433.

[FKK98] Ulrich Faigle, Walter Kern, and Jeroen Kuipers. "Note computing the nucleolus of min-cost spanning tree games is NP-hard". In: International Journal of Game Theory 27.3 (1998), pp. 443450.

[FKP00] Ulrich Faigle, Walter Kern, and Daniël Paulusma. "Note on the computational complexity of least core concepts for min-cost spanning tree games". In: Mathematical methods of operations research 52.1 (2000), pp. 23-38.

[GGZ98] Daniel Granot, Frieda Granot, and Weiping R Zhu. "Characterization sets for the nucleolus". In: International Journal of Game Theory 27.3 (1998), pp. 359-374.

[Gon85] Teofilo F Gonzalez. "Clustering to minimize the maximum intercluster distance". In: Theoretical computer science 38 (1985), pp. 293-306.

[Gra+96] Daniel Granot, Michael Maschler, Guillermo Owen, and Weiping R Zhu. "The kernel/nucleolus of a standard tree game". In: International Journal of Game Theory 25.2 (1996), pp. 219-244.

[Har17] John Hardwick. "Graphical Algorithms for Finding the Nucleolus of Binary-Valued Matching Games". PhD thesis. University of Illinois at Chicago, 2017.

[Kha79] Leonid Genrikhovich Khachiyan. "A polynomial algorithm in linear programming". In: Doklady Akademii Nauk. Vol. 244. 5. Russian Academy of Sciences. 1979, pp. 1093-1096.

[Kop67] Alexander Kopelowitz. Computation of the kernels of simple games and the nucleolus of n-person games. Tech. rep. Hebrew University of Jerusalem (Israel), Department of Mathematics, 1967.

[KP03] Walter Kern and Daniël Paulusma. "Matching games: the least core and the nucleolus". In: Mathematics of operations research 28.2 (2003), pp. 294-308.

[KPT20] Jochen Könemann, Kanstantsin Pashkovich, and Justin Toth. "Computing the nucleolus of weighted cooperative matching games in polynomial time". In: Mathematical Programming (2020), pp. $1-27$.

[KSA00] Jeroen Kuipers, Tamás Solymosi, and Harry Aarts. "Computing the nucleolus of some combinatoriallystructured games". In: Mathematical Programming 88.3 (2000), pp. 541-563.

[KT20] Jochen Könemann and Justin Toth. "A general framework for computing the nucleolus via dynamic programming". In: International Symposium on Algorithmic Game Theory. Springer. 2020, pp. 307-321.

[Lem84] Jean Lemaire. "An application of game theory: cost allocation". In: ASTIN Bulletin: The Journal of the IAA 14.1 (1984), pp. 61-81.

[MPS79] Michael Maschler, Bezalel Peleg, and Lloyd S Shapley. "Geometric properties of the kernel, nucleolus, and related solution concepts". In: Mathematics of operations research 4.4 (1979), pp. 303-338.

[NJ50] John F Nash Jr. "The bargaining problem". In: Econometrica: Journal of the econometric society (1950), pp. 155-162.

[Pau01] Daniël Paulusma. Complexity aspects of cooperative games. Citeseer, 2001. 
[Ple84] Ján Plesník. "A note on the complexity of finding regular subgraphs". In: Discrete mathematics 49.2 (1984), pp. 161-167.

[PRB06] Jos Potters, Hans Reijnierse, and Amit Biswas. "The nucleolus of balanced simple flow networks". In: Games and Economic Behavior 54.1 (2006), pp. 205-225.

[Sch03] Alexander Schrijver. Combinatorial optimization: polyhedra and efficiency. Vol. 24. Springer Science \& Business Media, 2003.

[Sch69] David Schmeidler. "The nucleolus of a characteristic function game". In: SIAM Journal on applied mathematics 17.6 (1969), pp. 1163-1170.

[SFS17] Balázs Sziklai, Tamás Fleiner, and Tamás Solymosi. "On the core and nucleolus of directed acyclic graph games". In: Mathematical Programming 163.1-2 (2017), pp. 243-271.

[SR94] Tamás Solymosi and Tirukkannamangai ES Raghavan. "An algorithm for finding the nucleolus of assignment games". In: International Journal of Game Theory 23.2 (1994), pp. 119-143.

[SS16] Tamás Solymosi and Balázs Sziklai. "Characterization sets for the nucleolus in balanced games". In: Operations Research Letters 44.4 (2016), pp. 520-524.

[SS71] Lloyd S Shapley and Martin Shubik. "The assignment game I: The core". In: International Journal of game theory 1.1 (1971), pp. 111-130.

[Ste68] Richard Edwin Stearns. "Convergent transfer schemes for n-person games". In: Transactions of the American Mathematical Society 134.3 (1968), pp. 449-459.

[Wil99] David Willer. Network exchange theory. Greenwood Publishing Group, 1999.

\section{Appendix}

\section{List of Figures}

1 The gadget graph from $[\operatorname{Bir}+18] \ldots \ldots \ldots \ldots \ldots \ldots$

2 A subgraph of $G_{1}$ for $k=2$, depicting the changes in Step I. . . . . . . . . . . . . . 12

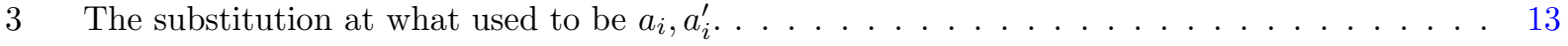

4 An illustration of Lemma 2.5 with $r=3, \kappa=2$ and $\Delta_{V^{\prime}}=4 \ldots \ldots \ldots \ldots$

\section{List of Tables}

1 The excess computation for Lemma 2.3 when $x^{*} \equiv \frac{3}{2}, S \subsetneq V_{u} \ldots \ldots \ldots \ldots \ldots$

2 The excess computation for Lemma 2.4 when $x_{\delta}, S \subsetneq V_{u} \ldots \ldots \ldots \ldots \ldots$ 